\title{
A Characterization of the Ordered Weighted Averaging Functions Based on the Ordered Bisymmetry Property
}

\author{
Jean-Luc Marichal* Pierre Mathonet $^{\dagger}$ \\ Revised version, September 28, 1997
}

\begin{abstract}
This paper deals with a characterization of a class of aggregation operators. This class concerns operators which are symmetric, increasing, stable for the same positive linear transformations and present a property close to the bisymmetry property: the ordered bisymmetry property. It is proved that the class investigated contains exactly the ordered weighted averaging operators (OWA) introduced by Yager in 1988.
\end{abstract}

Keywords: aggregation operators; interval scales; invariance; ordered bisymmetry property; ordered weighted averaging operators.

\section{Introduction}

Synthesizing judgments is an important part of multiple criteria decision making methods. The typical situation concerns individuals which form quantifiable judgments about a measure of an object (weight, length, area, height, volume, importance or other attributes, for instance in the framework of a hierarchy). In order to obtain a consensus of these jugdments, classical operators have been proposed: arithmetic means, root-power means, quasi-arithmetic means, fuzzy integrals and among them the ordered weighted averaging operators (OWA).

The OWA operators were proposed by Yager [9] and their links with fuzzy integrals were investigated by Grabisch [3, 4], and also by Fodor and Roubens [7, pages 135-140].

In this paper, we characterize the OWA operators under the natural properties of symmetry, increasing monotonicity, stability for the same interval scales in the sense of the theory of measurement (see [8]), but also under some kind of weak bisymmetry called "ordered bisymmetry" or "bisymmetry for ordered values". The bisymmetry property was studied in its initial form by Aczél [1, 2] (see also Fodor and Marichal [5]).

${ }^{*}$ Ecole d'Administration des Affaires, Université de Liège, Boulevard du Rectorat 7 - B31, B-4000 Liège, Belgium. E-mail: jl.marichal[at]ulg.ac.be

${ }^{\dagger}$ Institut de Mathématique, Université de Liège, Grande Traverse 12 - B37, B-4000 Liège, Belgium. E-mail: p.mathonet[at]ulg.ac.be 


\section{Basic definitions}

We first give the definition of an aggregation operator.

Definition 1 An aggregation operator $M$ defined on $\bigcup_{m \in \mathbb{N}_{0}} \mathbb{R}^{m}$ is a sequence $\left(M^{(m)}\right)_{m \in \mathbb{N}_{0}}$ of (aggregation) functions $M^{(m)}: \mathbb{R}^{m} \rightarrow \mathbb{R}$.

In order to obtain a reasonable or satisfactory aggregation, any aggregation operator could not be used: we have to impose that they must fulfil some properties. The next definition deals with this issue.

Definition 2 The aggregation operator $M$ defined on $\bigcup_{m \in \mathbb{N}_{0}} \mathbb{R}^{m}$ is

- symmetric (Sy) if, for all $m \in \mathbb{N}_{0}, M^{(m)}$ is a symmetric function on $\mathbb{R}^{m}$, i.e. if, for all $\left(x_{1}, \ldots, x_{m}\right) \in \mathbb{R}^{m}$ and all permutation $\sigma$ of $\{1, \ldots, m\}$,

$$
M^{(m)}\left(x_{1}, \ldots, x_{m}\right)=M^{(m)}\left(x_{\sigma(1)} \ldots, x_{\sigma(m)}\right) .
$$

- increasing (In) if, for all $m \in \mathbb{N}_{0}, M^{(m)}$ is increasing on each argument, i.e. if, for all $i \in\{1, \ldots, m\}$ and all $x_{1}, \ldots, x_{m}, x_{i}^{\prime} \in \mathbb{R}$, we have

$$
x_{i}<x_{i}^{\prime} \Rightarrow M^{(m)}\left(x_{1}, \ldots, x_{i}, \ldots, x_{m}\right) \leq M^{(m)}\left(x_{1}, \ldots, x_{i}^{\prime}, \ldots, x_{m}\right) .
$$

- stable for the same positive linear transformations (SPL) if, for all $m \in \mathbb{N}_{0}$, we have

$$
M^{(m)}\left(r x_{1}+t, \ldots, r x_{m}+t\right)=r M^{(m)}\left(x_{1}, \ldots, x_{m}\right)+t
$$

for all $\left(x_{1}, \ldots, x_{m}\right) \in \mathbb{R}^{m}$, all $r>0$ and all $t \in \mathbb{R}$.

- bisymmetric for ordered values $(\mathrm{OB})$ if $M^{(1)}(x)=x$ for all $x \in \mathbb{R}$ and if, for all $m, p \in \mathbb{N}_{0}$,

$$
\begin{aligned}
& M^{(p)}\left(M^{(m)}\left(x_{11}, \ldots, x_{1 m}\right), \ldots, M^{(m)}\left(x_{p 1}, \ldots, x_{p m}\right)\right) \\
= & M^{(m)}\left(M^{(p)}\left(x_{11}, \ldots, x_{p 1}\right), \ldots, M^{(p)}\left(x_{1 m}, \ldots, x_{p m}\right)\right)
\end{aligned}
$$

for all ordered matrices

$$
X=\left(\begin{array}{ccc}
x_{11} & \cdots & x_{1 m} \\
\vdots & & \vdots \\
x_{p 1} & \cdots & x_{p m}
\end{array}\right) \in \mathbb{R}^{p \times m}
$$

where $X$ is ordered if its elements satisfy $x_{i j} \leq x_{k l}$ whenever $i \leq k$ and $j \leq l$.

The first three properties (Sy, In, SPL) are well-known and seem natural enough (see e.g. [7]). The (OB) property is a weak form of the algebraic property "bisymmetry" which expresses that aggregation can be performed first on the rows, then on the columns of any matrix, or conversely (see $[1,2])$. We introduce it in this paper for our needs. 
Justification of the bisymmetry property: Suppose that $m$ judges give a score (real number) to each of $p$ candidates. We could put these numbers in a $p \times m$ matrix as follows:

$$
\begin{gathered}
J_{1} \\
C_{1} \\
\vdots \\
C_{p}
\end{gathered}\left(\begin{array}{ccc}
x_{11} & \cdots & x_{m} \\
\vdots & & \vdots \\
x_{p 1} & \cdots & x_{p m}
\end{array}\right)
$$

Suppose now that we want a single value reflecting the global score of the $p$ candidates. A natural answer would be to compute the arithmetic mean of the values in the table. But there are some cases where the arithmetic mean is no longer convenient - for example when the judges are supposed to have some weights. Another answer would be the following: aggregation of the scores of each candidate (aggregation on the rows of the matrix), and then aggregation of these global values. But there is another way: aggregation of the scores given by each judge (aggregation on the columns of the matrix), and then aggregation of these values. The classical bisymmetry property for an aggregation function tells that we can choose either the first or the second manner to proceed; the result will be the same. This is a rather natural property.

Justification of the ordered bisymmetry property: Suppose that we are in the same situation as hereabove: $p$ candidates and $m$ judges provinding a matrix of appraisals. But we start by removing some values - for example the lowest scores given by each judge and the lowest scores obtained by each candidate. In general, it does not make sense anymore to aggregate first on the rows and then on the columns, or conversely, and to ask to obtain the same global evaluation: indeed, doing so, we do not aggregate the same values in the matrix.

Now there is a situation where it still make sense: if the worst candidate is the same for each judge and the hardest judge is the same for each candidate, when removing them, we get a score matrix for $(p-1)$ candidates and $(m-1)$ judges, and we can aggregate as before. Remark that this situation correspond to a certain order in the matrix $X$. If we wish to take into account all the possibilities to remove one or several judges and candidates, we have to consider orderable matrices of scores. Under the symmetry assuption (Sy), this is equivalent to consider ordered matrices. In this context, ordered bisymmetry property is then the classical bisymmetry property, after removing some values and when it still makes sense.

\section{Ordered weighted averaging functions}

Now, we can introduce the ordered weighted averaging operators.

Definition $3(i)$ Let $m \in \mathbb{N}_{0}$. For any weight vector $\omega^{(m)}=\left(\omega_{1}^{(m)}, \ldots, \omega_{m}^{(m)}\right) \in[0,1]^{m}$ such that

$$
\sum_{i=1}^{m} \omega_{i}^{(m)}=1
$$

the ordered weighted averaging function $O W A_{\omega^{(m)}}^{(m)}$ defined on $\mathbb{R}^{m}$ and associated to $\omega^{(m)}$ is defined by

$$
O W A_{\omega^{(m)}}^{(m)}\left(x_{1}, \ldots, x_{m}\right)=\sum_{i=1}^{m} \omega_{i}^{(m)} x_{(i)}, \quad \forall\left(x_{1}, \ldots, x_{m}\right) \in \mathbb{R}^{m}
$$


where $x_{1}, \ldots, x_{m}$ are reordered as $x_{(1)} \leq \ldots \leq x_{(i)} \leq \ldots \leq x_{(m)}$.

(ii) For any sequence $\omega=\left(\omega^{(m)}\right)_{m \in \mathbb{N}_{0}}$ of weight vectors $\omega^{(m)} \in[0,1]^{m}$, such that

$$
\sum_{i=1}^{m} \omega_{i}^{(m)}=1
$$

the ordered weighted averaging operator $O W A_{\omega}$ defined on $\bigcup_{m \in \mathbb{N}_{0}} \mathbb{R}^{m}$ and associated to $\omega$ is the operator $\left(O W A_{\omega(m)}^{(m)}\right)_{m \in \mathbb{N}_{0}}$.

\section{Characterization of the OWA functions}

Before obtaining the announced characterization, we first need some technical lemmas.

Lemma 1 If the aggregation function $M$ defined on $\bigcup_{m \in \mathbb{N}_{0}} \mathbb{R}^{m}$ satisfies (In) and (SPL), then

- $M$ is idempotent, i.e. for all $m \in \mathbb{N}_{0}$,

$$
M^{(m)}(x, \ldots, x)=x
$$

for all $x \in \mathbb{R}$.

- $M$ is compensative, i.e. for all $m \in \mathbb{N}_{0}$,

$$
\min _{i} x_{i} \leq M^{(m)}\left(x_{1}, \ldots, x_{m}\right) \leq \max _{i} x_{i}
$$

for all $x_{1}, \ldots, x_{m} \in \mathbb{R}$.

Proof. From (SPL) we have

$$
M^{(m)}(0, \ldots, 0)=r M^{(m)}(0, \ldots, 0)
$$

for all $r>0$, and hence $M^{(m)}(0, \ldots, 0)=0$. We also have $M^{(m)}(x, \ldots, x)=M^{(m)}(0, \ldots, 0)+$ $x=x \forall x \in \mathbb{R}$. Finally, from (In) we have

$$
M^{(m)}\left(\min _{i} x_{i}, \ldots, \min _{i} x_{i}\right) \leq M^{(m)}\left(x_{1}, \ldots, x_{m}\right) \leq M^{(m)}\left(\max _{i} x_{i}, \ldots, \max _{i} x_{i}\right) .
$$

It turns out, by the previous lemma, that the condition " $r>0$ " in the statement of the (SPL) property can be replaced by " $r \geq 0$ " without change.

Let $m \in \mathbb{N}_{0}$. For all $i \in\{0, \ldots, m\}$, let us introduce

$$
\theta_{i}^{(m)}=M^{(m)}(\underbrace{0, \ldots, 0}_{i}, 1 \ldots, 1) .
$$

Under the assumptions of Lemma 1 , we have $\theta_{i}^{(m)} \in[0,1] \forall i \in\{0, \ldots, m\}$. We then have the following lemma. 
Lemma 2 Let $M$ be an aggregation operator defined on $\bigcup_{m \in \mathbb{N}_{0}} \mathbb{R}^{m}$ and satisfying (In), $(S P L)$ and $(O B)$. Let $m \in \mathbb{N}_{0}$. If there exists $k \in\{1, \ldots, m\}$ such that $\theta_{k}^{(m)} \in(0,1)$ then $M^{(m)}$ is additive for ordered values, i.e.

$$
M^{(m)}\left(u_{1}+v_{1}, \ldots, u_{m}+v_{m}\right)=M^{(m)}\left(u_{1}, \ldots, u_{m}\right)+M^{(m)}\left(v_{1}, \ldots, v_{m}\right)
$$

for all $\left(u_{1}, \ldots, u_{m}\right),\left(v_{1}, \ldots, v_{m}\right) \in \mathbb{R}^{m}$ such that $u_{1} \leq \ldots \leq u_{m}$ and $v_{1} \leq \ldots \leq v_{m}$.

Proof. Let $\left(u_{1}, \ldots, u_{m}\right),\left(v_{1}, \ldots, v_{m}\right) \in \mathbb{R}^{m}$ such that $u_{1} \leq \ldots \leq u_{m}$ and $v_{1} \leq \ldots \leq v_{m}$.

(i) Assume first that $u_{1}, v_{1} \geq 0$. Consider the ordered square matrix $X$ of $m$ rows $r_{i}$ and $m$ columns $c_{j}(i, j=1, \ldots, m)$, where $r_{i}$ is defined as follows $($ for $i=1, \ldots, m)$ :

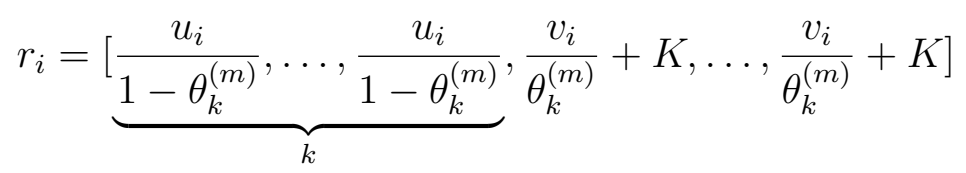

with $K=\frac{u_{m}}{1-\theta_{k}^{(m)}}$. On the one hand, by (SPL), we have, for all $i \in\{1, \ldots, m\}$ :

$$
M^{(m)}\left(r_{i}\right)=\frac{u_{i}}{1-\theta_{k}^{(m)}}+\left(\frac{v_{i}}{\theta_{k}^{(m)}}+K-\frac{u_{i}}{1-\theta_{k}^{(m)}}\right) \theta_{k}^{(m)}=u_{i}+v_{i}+K \theta_{k}^{(m)},
$$

and thus

$$
M^{(m)}\left(M^{(m)}\left(r_{1}\right), \ldots, M^{(m)}\left(r_{m}\right)\right)=M^{(m)}\left(u_{1}+v_{1}, \ldots, u_{m}+v_{m}\right)+K \theta_{k}^{(m)} .
$$

On the other hand, for all $j \in\{1, \ldots, m\}$, we have

$$
M^{(m)}\left(c_{j}\right)= \begin{cases}\frac{M^{(m)}\left(u_{1}, \ldots, u_{m}\right)}{1-\theta_{k}^{(m)}} & \text { if } j \leq k \\ \frac{M^{(m)}\left(v_{1}, \ldots, v_{m}\right)}{\theta_{k}^{(m)}}+K & \text { otherwise. }\end{cases}
$$

However, by Lemma 1 , we have $M^{(m)}\left(u_{1}, \ldots, u_{m}\right) \leq u_{m}$ and $M^{(m)}\left(v_{1}, \ldots, v_{m}\right) \geq 0$. Hence, by (SPL), we have

$$
\begin{aligned}
& M^{(m)}\left(M^{(m)}\left(c_{1}\right), \ldots, M^{(m)}\left(c_{m}\right)\right) \\
= & \frac{M^{(m)}\left(u_{1}, \ldots, u_{m}\right)}{1-\theta_{k}^{(m)}}+\left(\frac{M^{(m)}\left(v_{1}, \ldots, v_{m}\right)}{\theta_{k}^{(m)}}+K-\frac{M^{(m)}\left(u_{1}, \ldots, u_{m}\right)}{1-\theta_{k}^{(m)}}\right) \theta_{k}^{(m)} \\
= & M^{(m)}\left(u_{1}, \ldots, u_{m}\right)+M^{(m)}\left(v_{1}, \ldots, v_{m}\right)+K \theta_{k}^{(m)} .
\end{aligned}
$$

Since $M^{(m)}$ fulfils (OB), we have

$$
M^{(m)}\left(u_{1}+v_{1}, \ldots, u_{m}+v_{m}\right)=M^{(m)}\left(u_{1}, \ldots, u_{m}\right)+M^{(m)}\left(v_{1}, \ldots, v_{m}\right)
$$

whenever $0 \leq u_{1} \leq \ldots \leq u_{m}$ and $0 \leq v_{1} \leq \ldots \leq v_{m}$.

(ii) In the general case, we have, using $(i)$ and (SPL),

$$
\begin{aligned}
& M^{(m)}\left(u_{1}+v_{1}, \ldots, u_{m}+v_{m}\right) \\
= & M^{(m)}\left(0,\left(u_{2}-u_{1}\right)+\left(v_{2}-v_{1}\right), \ldots,\left(u_{m}-u_{1}\right)+\left(v_{m}-v_{1}\right)\right)+u_{1}+v_{1} \\
= & M^{(m)}\left(0, u_{2}-u_{1}, \ldots, u_{m}-u_{1}\right)+u_{1}+M^{(m)}\left(0, v_{2}-v_{1}, \ldots, v_{m}-v_{1}\right)+v_{1} \\
= & M^{(m)}\left(u_{1}, \ldots, u_{m}\right)+M^{(m)}\left(v_{1}, \ldots, v_{m}\right) .
\end{aligned}
$$

Now, let us turn to the announced characterization. 
Theorem 1 The aggregation operator $M$ defined on $\bigcup_{m \in \mathbb{N}_{0}} \mathbb{R}^{m}$ fulfils (Sy), (In), (SPL) and $(O B)$ if and only if, for all $m \in \mathbb{N}_{0}$, there exists $\omega^{(m)} \in[0,1]^{m}$ such that

$$
M^{(m)}=O W A_{\omega(m)}^{(m)} .
$$

Proof. (Sufficiency). We can easily check that any aggregation operator satisfies the (Sy), (In), (SPL) and (OB) properties.

(Necessity). Fix $m \in \mathbb{N}_{0}$ and let $\left(x_{1}, \ldots, x_{m}\right) \in \mathbb{R}^{m}$. We have $M^{(m)}\left(x_{1}, \ldots, x_{m}\right) \stackrel{(S y)}{=} M^{(m)}\left(x_{(1)}, \ldots, x_{(m)}\right) \stackrel{(S P L)}{=} M^{(m)}\left(0, x_{(2)}-x_{(1)}, \ldots, x_{(m)}-x_{(1)}\right)+x_{(1)}$, with $x_{(1)} \leq \ldots \leq x_{(m)}$. We obviously have two exclusive cases:

( $i$ ) If there exists $k \in\{1, \ldots, m\}$ such that $\theta_{k}^{(m)} \in(0,1)$ then, from Lemma $2, M^{(m)}$ is additive for ordered values. We have, for all $i \in\{2, \ldots, m\}$,

$$
\begin{aligned}
& M^{(m)}(\underbrace{0, \ldots, 0}_{i-1}, x_{(i)}-x_{(i-1)}, \ldots, x_{(m)}-x_{(i-1)}) \\
= & M^{(m)}(\underbrace{0, \ldots, 0}_{i-1}, x_{(i)}-x_{(i-1)}, \ldots, x_{(i)}-x_{(i-1)})+M^{(m)}(\underbrace{0, \ldots, 0}_{i}, x_{(i+1)}-x_{(i)}, \ldots, x_{(m)}-x_{(i)}) \\
= & \left(x_{(i)}-x_{(i-1)}\right) \theta_{i-1}^{(m)}+M^{(m)}(\underbrace{0, \ldots, 0}_{i}, x_{(i+1)}-x_{(i)}, \ldots, x_{(m)}-x_{(i)})
\end{aligned}
$$

and thus, recursively,

$$
M^{(m)}\left(x_{1}, \ldots, x_{m}\right)=\sum_{i=2}^{m}\left(x_{(i)}-x_{(i-1)}\right) \theta_{i-1}^{(m)}+x_{(1)}=\sum_{i=1}^{m}\left(\theta_{i-1}^{(m)}-\theta_{i}^{(m)}\right) x_{(i)} .
$$

By (In), $\theta_{i-1}^{(m)}-\theta_{i}^{(m)} \geq 0$ for all $i \in\{1, \ldots, m\}$. Moreover,

$$
\sum_{i=1}^{m}\left(\theta_{i-1}^{(m)}-\theta_{i}^{(m)}\right)=\theta_{0}^{(m)}-\theta_{m}^{(m)}=1-0=1
$$

as required.

(ii) If there exists $k \in\{1, \ldots, m\}$ such that

$$
\theta_{i}^{(m)}= \begin{cases}0 & \forall i \in\{k, \ldots, m\} \\ 1 & \forall i \in\{1, \ldots, k-1\}\end{cases}
$$

then, by (In) and (SPL), we have,

$$
\begin{aligned}
M^{(m)}\left(x_{(1)}, \ldots, x_{(m)}\right) & \leq M^{(m)}(\underbrace{x_{(k)}, \ldots, x_{(k)}}_{k}, x_{(m)}, \ldots, x_{(m)}) \\
& =x_{(k)}+M^{(m)}(\underbrace{0, \ldots, 0}_{k}, x_{(m)}-x_{(k)}, \ldots, x_{(m)}-x_{(k)}) \\
& =x_{(k)}
\end{aligned}
$$

and

$$
\begin{aligned}
M^{(m)}\left(x_{(1)}, \ldots, x_{(m)}\right) & \geq M^{(m)}(\underbrace{x_{(1)}, \ldots, x_{(1)}}_{k-1}, x_{(k)}, \ldots, x_{(k)}) \\
& =M^{(m)}(\underbrace{0, \ldots, 0}_{k-1}, x_{(k)}-x_{(1)}, \ldots, x_{(k)}-x_{(1)})+x_{(1)} \\
& =x_{(k)}-x_{(1)}+x_{(1)} \\
& =x_{(k)} .
\end{aligned}
$$


Thus, we have $M^{(m)}\left(x_{(1)}, \ldots, x_{(m)}\right)=x_{(k)}$ and

$$
M^{(m)}=\mathrm{OWA}_{\omega(m)}^{(m)}
$$

with $\omega_{k}^{(m)}=1$.

\section{References}

[1] J. Aczél (1948), On mean values, Bulletin of the American Math. Society, 54 : 392-400.

[2] J. Aczél (1966), Lectures on Functional Equations and Applications, (Academic Press, New-York).

[3] M. Grabisch (1993), On the use of fuzzy integral as a fuzzy connective, Proceedings of the Second IEEE International Conference on Fuzzy Systems, San Francisco : 213-218.

[4] M. Grabisch (1995), On equivalence classes of fuzzy connectives : the case of fuzzy integrals, IEEE Trans. Fuzzy Syst., vol. 3, no. 1 : 96-109.

[5] J. Fodor and J.-L. Marichal (1997), On nonstrict means, Aequationes Mathematicae 54: $308-327$.

[6] J. Fodor, J.-L. Marichal and M. Roubens (1994), Characterization of some aggregation functions arising from MCDM problems, Proceedings of IPMU'94, Paris: 1026-1031.

[7] J. Fodor and M. Roubens (1994), Fuzzy Preference Modelling and Multicriteria Decision Support, Kluwer, Dordrecht.

[8] F.S. Roberts (1979), Measurement Theory with Applications to Decision-making, Utility and the Social Sciences, (Addison-Wesley Pub., Reading).

[9] R.R. Yager (1988), On Ordered Weighted Averaging Aggregation Operators in Multicriteria Decision making, IEEE Trans. on Systems, Man and Cybernetics, 18 : 183-190. 